\title{
DESARROLLO DE UN SISTEMA EMBEBIDO BASADO EN LÓGICA DIFUSA PARA EL RECONOCIMIENTO DEL ESTADO DE EBRIEDAD EN CONDUCTORES ORIENTADO AL BLOQUEO DEL ARRANQUE DE UN VEHÍCULO
}

\author{
Development of an embedded system based on diffuse logic to identify a \\ driver drunkenness level in order to block his/her car ignition start
}

\author{
Enrique Jesús Martínez Amaya (201310461) \\ José Luis Ferreyra Ramos (201411678)2 \\ Brayan Ramírez Mejía (201310457)
}

\begin{abstract}
RESUMEN
El presente trabajo de investigación consiste en el estudio y diseño de un controlador difuso para detectar el posible estado de ebriedad en conductores de empresas vehiculares y evitar que estos pongan en riesgo su vida o la de otras personas.

Este proyecto de investigación se basa en el hecho de que existen en el mercado nacional sensores que permiten detectar la concentración de alcohol en el aire, así como para la medida del peso corporal. De la misma manera, en la actualidad, existen software como el Labview, Matlab, Phyton, entre otros, en los que es viable la implementación de algoritmos de acondicionamiento de señales y de controladores difusos, los cuales resultan de mucha utilidad para el sistema planteado. Por lo tanto, la implementación de este proyecto de investigación se justifica porque todo el hardware y software a utilizarse se encuentra al alcance del investigador.
\end{abstract}

\begin{abstract}
This research work consists in the study and design of a diffuse controller to detect the possible influence of alcohol in drivers of vehicle companies and avoid that it can handle in this State.

This research project is justified by finding sensors that can detect the concentration of alcohol in the air in the domestic market, as well as the existence of sensors for the measurement of body weight. In the same way, at present, there are software such as Labview, Matlab, Python, among others, where the implementation of algorithms of conditioning of signals and fuzzy controllers, which are very useful for the proposed system is viable. Therefore, this research project has justification be implemented because all the hardware and software to be used, is available to the researcher.
\end{abstract}

1 Escuela Profesional de Ingeniería Electrónica, Universidad Ricardo Palma. E-mail: enrike.electronix7@gmail.com

2 Escuela Profesional de Ingeniería Electrónica, Universidad Ricardo Palma. E-mail: jferreyra2415@gmail.com

3 Escuela Profesional de Ingeniería Electrónica, Universidad Ricardo Palma. E-mail: brayan_23_96@hotmail.com 


\section{INTRODUCCIÓN}

En esta tesis se plantea la siguiente pregunta. ¿Es posible implementar un prototipo de control difuso, en un sistema embebido Raspberry Pi 3, para diagnosticar conductores de vehículos en estado de posible ebriedad? Para este fin tenemos como principal objetivo implementar un prototipo de control difuso, en un sistema embebido Raspberry Pi 3, para determinar si el conductor de un vehículo se encuentra en un posible estado de ebriedad. Asimismo, implementar un sistema el cual permita la inhabilitación de la marcha del vehículo, además de generar una señal sonora y/o visual. La presente investigación se enfocará en el estudio de la lógica difusa para la detección del estado de ebriedad en los conductores con el fin de controlar las dos principales variables de este proceso, el peso y los gramos de alcohol en la sangre. Esta investigación se justifica debido a la gran cantidad de accidentes producidos por conductores en estado de ebriedad siendo que este prototipo puede ayudar a disminuir esta cantidad de accidentes. En los capítulos desarrollados se describirán los principales aspectos desarrollados para esta tesis, siendo que en el capítulo se formula el problema y los objetivos de esta investigación. En el capítulo dos se desarrolla los términos teóricos importantes además de revisar los antecedentes a este proyecto. En el tercero se describe la mitología de investigación, haciendo énfasis en las técnicas de recolección de datos y el estudio y la población de estudio. En el cuarto se realiza la descripción del diseño del proyecto, tanto de los diagramas electrónicos como de la implementación del prototipo final. En el capítulo cinco se realiza la evaluación del sistema mostrando y analizando los datos de las diferentes pruebas realizadas para comprobar la funcionalidad y eficiencia del prototipo. Finalmente se muestran las conclusiones generales de todo el proyecto.

\section{PRESENTACIÓN DEL PROBLEMA}

Existe una gran cantidad de accidentes automovilísticos en las carreteras peruanas, los cuales se buscan reducir aumentando el control policial de tránsito; sin embargo, esto no es suficiente para brindar una seguridad óptima a los transeúntes.

\section{SOLUCIÓN}

El presente proyecto busca implementar como solución a un problema social un sistema de automatización. Por ello se decidió desarrollar un sistema de detección de estado etílico en conductores mediante algoritmos difusos, con el fin de evitar de que algunas personas conduzcan en estado de ebriedad.

\section{DISEÑO DEL PROYECTO DE INVESTIGACIÓN}

El diseño del proyecto se llevó a cabo en diferentes etapas, las cuales se detallan a continuación:

\subsection{Alimentación del sistema}

En los vehículos, la alimentación eléctrica es suministrada por una batería eléctrica cuyo voltaje, en el mayor de los casos, es $12 \mathrm{~V}$, razón por la cual será necesario utilizar un regulador de voltaje de $5 \mathrm{~V}$ para poder cumplir con las especificaciones de alimentación principalmente RapsBerry pi. 
El regulador planteado para este proyecto será diseñado con un LM317, el cual permitirá regular el voltaje de salida según sea lo deseado (en este caso, $5 \mathrm{~V}$ ), el cual se explicará con mayor detalle posteriormente. Además, será implementado en un circuito impreso el cual obtendrá su voltaje de entrada de la línea de ACC del conmutador. De esta forma, todo el sistema planteado se alimentará cuando se ponga en la primera posición la llave.

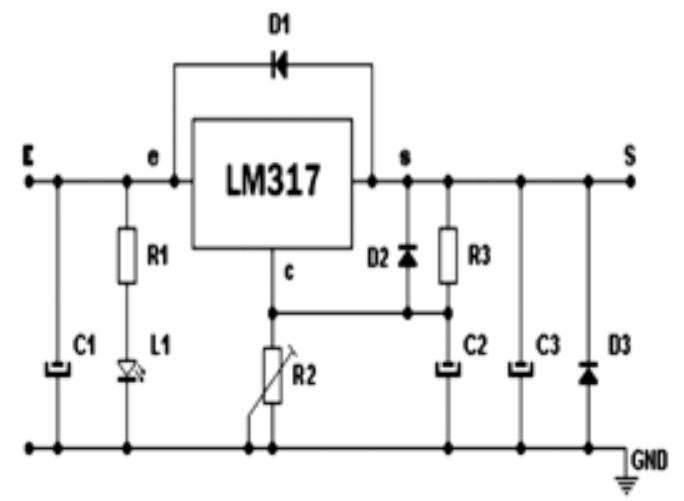

Fig. 1. Regulador de voltaje propuesto por el fabricante [2]

\subsection{Circuito de activación del sistema}

Como se describió anteriormente, parte fundamental del proyecto está en la inhabilitación del encendido del motor. Para ello, nos basamos en un diagrama típico de arranque vehicular el cual se muestra en la fig. 2. En este diagrama, observamos que el relé es activado cuando el interruptor es encendido y activa la línea azul. De esta forma, cierra el circuito entre la batería y el motor del vehículo.

Haciendo uso de este concepto, utilizaremos una condición de activación adicional, la cual será accionada por el Raspberry después de analizar las variables de alcohol y peso obtenidos de los sensores en el algoritmo difuso. En el caso de que los parámetros estén dentro de lo permitido para la conducción, el Raspberry mandará un pulso de activación a un relé secundario el cual se comportara como circuito abierto en la línea de START conmutador de arranque, que es la condición inicial del vehículo para ponerse en marcha. En caso contrario, no se emitirá este pulso ocasionando que el relé secundario no cierre circuito y, por lo tanto, inhabilite la marcha del automóvil.

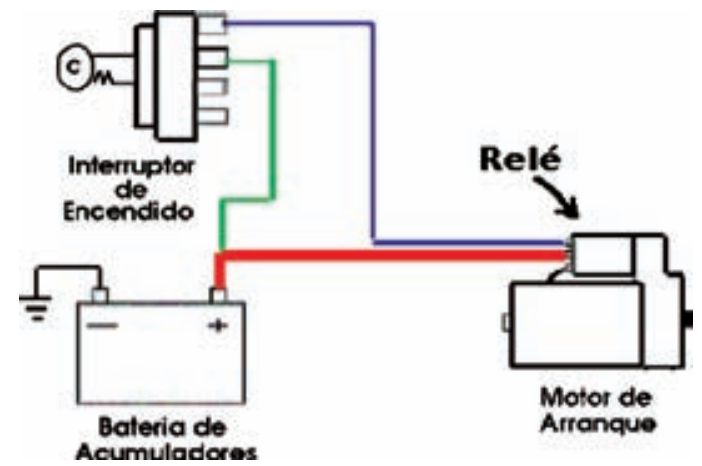

Fig. 2. Circuito de arranque vehicular [1] 


\subsection{Circuito de sensor MQ-3}

Este sistema acondiciona el nivel de voltaje que llega al sensor de gas mediante un divisor de tensión entre la resistencia interna del sensor y una resistencia externa, en este caso de $10 \mathrm{~K}$ ohm. Esto se realiza con la finalidad de calibrar la sensibilidad del sensor. Adicionalmente, se utiliza un regulador de voltaje 7805 no regulable el cual brinda un voltaje de salida 5 v constante, esto para mayor protección según especificaciones técnicas del fabricante.

Debido a que la señal de salida es analógica, se opta por hacer uso de un ATMEGA328p como conversor analógico digital con la finalidad de enviar los datos medidos por el sensor al Raspberry para su análisis en el algoritmo difuso.

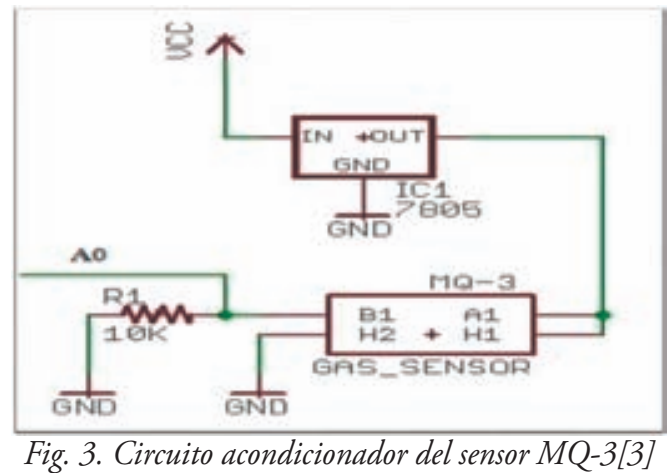

\subsection{Circuito de la celda de carga}

Para el acondicionamiento de la celda de carga, es necesario el uso de un amplificador de instrumentación (INA) debido a los bajos niveles de salida en sus terminales de señal (uno de señal negativa y otro de señal positiva), además de un conversor analógico digital (DAC) para que sea posible el procesamiento posterior por el Raspberry.

Este circuito de acondicionamiento, para mayor exactitud, es implementado haciendo uso de un circuito integrado HX711 especializado para este tipo de sensores, el cual tiene integrados un INA de bajo ruido y un DAC de 24 bits de resolución. Los complementos para este circuito se toman de las recomendaciones del fabricante. Es importante observar que se utilizan filtros paso bajo, los cuales sirven de acoplamiento para cada una de las señales de entrada $S-$ y $S_{+}$, como se muestra en la fig.4.

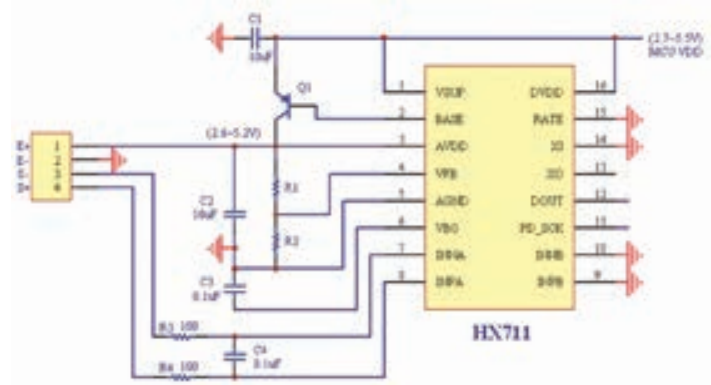

Fig. 4. Diagrama de acondicionamiento de la celda de carga con HX711 [4] 


\subsection{Controlador difuso}

Diseño del algoritmo difuso: Para el diseño del algoritmo difuso, se decidió aplicar la lógica difusa de tipo Mamdani en el control con el cual se obtendrá una respuesta final hacia nuestro sistema de arranque del vehículo.

Variables difusas de Entrada: Para nuestro controlador, se usó dos variables de entrada: Alcohol y Peso. Estas variables están compuestas por 3 conjuntos difusos ( 2 trapezoides y 1 triángulo). Cada conjunto está parametrizado de la forma más adecuada por un "experto" en el tema a tratar.

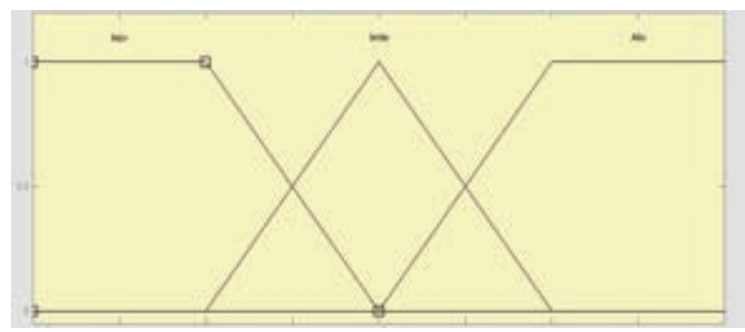

Fig. 5. Variable de nivel de sangre medido en la sangre

Variable de Alcohol: Esta variable es la encargada de establecer límites en los niveles de alcohol que debe tener una personar al conducir un vehículo. Estos límites están establecidos de acuerdo con el reglamento del límite máximo de alcohol en la sangre que puede tener un conductor en las carreteras peruanas.

Variable de Peso: Esta variable se encarga de establecer, de igual manera, los límites de peso de una persona que podría conducir un vehículo. Esta variable es de gran importancia en el proyecto al igual que la de alcohol, debido a que existe una relación científica sobre variación de la influencia del alcohol ingerido en personas de diferentes pesos corporales.

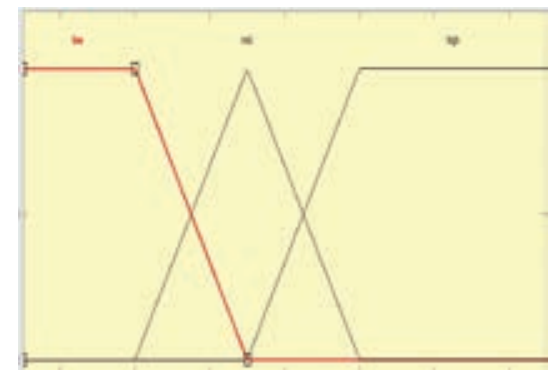

Fig. 6. Variable de peso promedio en los conductores de vehiculos en Perú

Variable difusa de Salida: El algoritmo difuso, además, lleva una variable de salida la cual nos brindará una referencia numérica de la acción a tomar según nuestras reglas planteadas en el controlador. Esta variable está diseñada con dos conjuntos difusos triangulares, 'Encendido' y 'Apagado'. El primero define el encendido y el segundo, la marcha del automóvil.

4 Experto: persona con conocimientos basados en investigaciones o experiencias propias acerca de cada variable que se use en el algoritmo. 


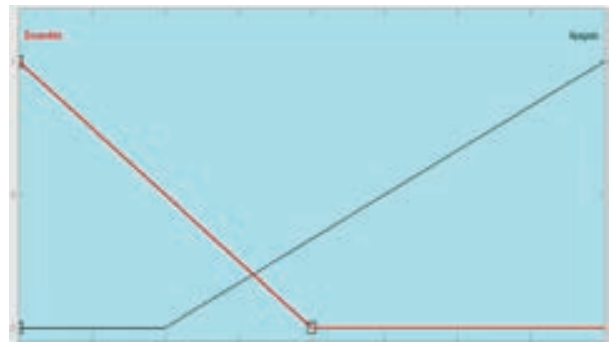

Fig. 7. Variable de salida del controlador Difuso

\section{DIAGRAMAS DE INGENIERÍA}

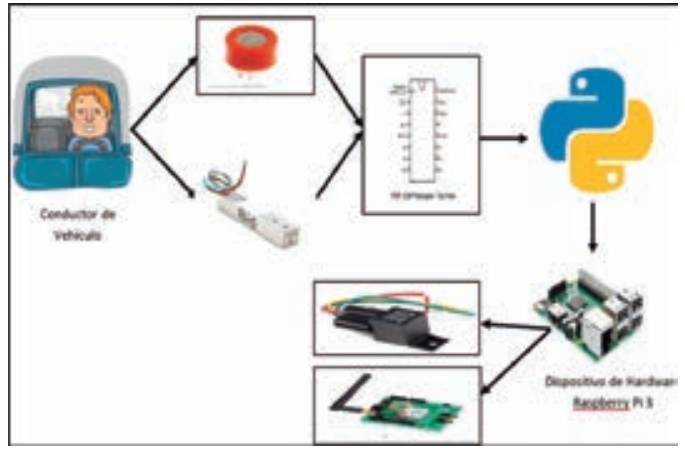

Fig.7. Diagrama pictórico del proyecto [5]

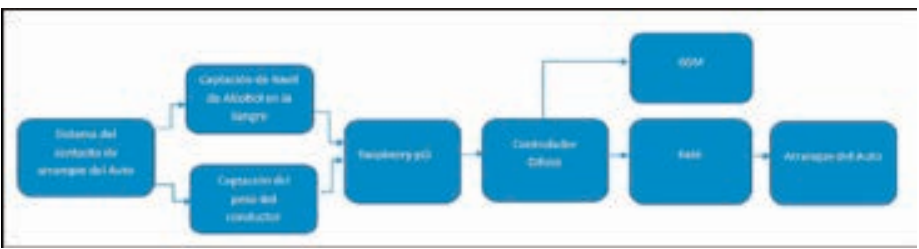

Fig. 8. Diagrama de bloques general del sistema [5]

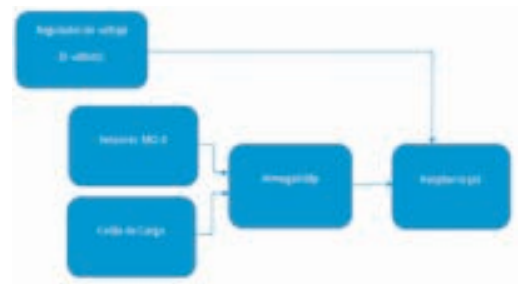

Fig. 9. Diagrama de bloques de las conexiones de entrada del Raspberry Pi [5] 


\section{RESULTADOS}

El sistema eléctrico que alimentara al controlador y demás elementos periféricos involucrados se encienden y funcionan de manera correcta sin que se presente ninguna caída de tensión repentina en el momento de tener el sistema en funcionamiento. Asimismo, hay una correcta lectura de los sensores: tanto de la celda de carga como también de los MQ-3 en el controlador Embebido Raspberry. Finalmente, el algoritmo difuso que se encargará de designar un valor de salida adecuado según sus niveles de lectura en las entradas tiene un funcionamiento correcto, pudiendo asegurar esto mediante su simulación en el GUI Fuzzy de Matlab.

\section{Análisis de resultados}

Análisis en el controlador Difuso: Para poder llevar acabo un correcto análisis acerca del funcionamiento de nuestro controlador difuso, antes de comprobar los valores proporcionados por la programación en código y el del GUI Fuzzy, se debe realizar un análisis aún más importante, el cual corresponde a la verificación de las reglas que se proporcionaron para el análisis del programa según nuestros estándares planteados en el proyecto. Para llevar a cabo esta verificación se hace uso de una tabla de reglas difusas.

Tabla 1. Tabla de Reglas Difusas

\begin{tabular}{|c|c|c|c|}
\hline $\begin{array}{l}\text { Alco } \\
\text { Peso }\end{array}$ & Bajo & Lim & Alto \\
\hline Low & Apagado & Apagado & Apagado \\
\hline Mid & Encendido & Apagado & Apagado \\
\hline High & Encendido & Encendido & Apagado \\
\hline
\end{tabular}

Finalmente, se realizó una simulación del algoritmo difuso en el lenguaje Python, el cual nos muestra los resultados observados en la fig. 22 en donde se observa que para una entrada de nivel bajo $(0.12 \mathrm{gr} /$ litro de alcohol) y una entrada de nivel Mid (peso de $72.7 \mathrm{Kg}$ ) se obtiene un valor de salida de 2.22, el cual corresponde al conjunto de salida 'Encendido' (ver fig. 7), estando acorde este resultado con lo propuesto en la Tabla 1.

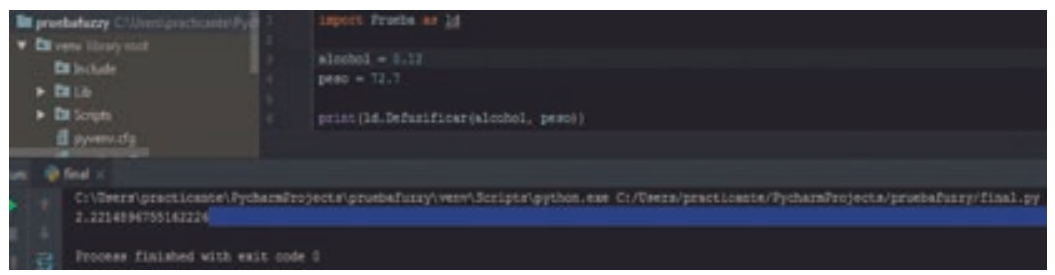

Fig. 10. Simulación de resultado del algoritmo difuso en Python para una respuesta en el conjunto 'Encendido'

Finalmente, para un nivel 'Alto' y peso 'Mid' se obtiene una salida de 4.033, la cual corresponde al conjunto 'Apagado' demostrando de esta forma la operatividad del algoritmo (Ver fig.11). 


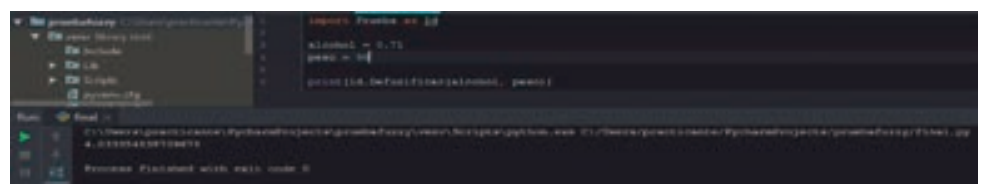

Fig.11. Simulación de resultado del algoritmo difuso en Python para una respuesta en el conjunto 'Apagado'

\section{CONCLUSIONES}

Del desarrollo de esta investigación podemos concluir que el diseño e implementación de un algoritmo difuso puede ser implementado en un Raspberry pi, lo cual fue corroborado mediante simulación en Python, con lo que se obtuvo los resultados esperados. De esta forma, se puede tomar la decisión de habilitar o inhabilitar la conducción vehicular en base a los datos obtenidos de los sensores (MQ3 y celda de carga). Además, podemos decir que el manejo del módulo GSM para el envío de mensajes de texto resulta de baja complejidad, por lo que esta parte se cumple efectivamente teniendo una alta importancia de comunicar el estado de ebriedad del conductor a alguien que pueda brindarle ayuda.

\section{REFERENCIAS}

[1] El motor de encendido del auto (parte 1), 2012. [En línea]. Disponible en: http://www.mecanicaymotores.com/el-interruptor-de-encendido-del-auto-parte-1.html. [Accedido: 2-jun-2018]

[2] Fuente de alimentación, 2018. Visitado en mayo 2018. [En línea]. Disponible en: http://perso. wanadoo.es/pictob/fuente.htm. [Accedido: 14-may-2018]

[3] Calibrating the MQ-3 Alcohol Sensor, 2108. [En línea]. Disponible en: http://nootropicdesign. com/projectlab/2010/09/17/arduino-breathalyzer/. [Accedido: 14-may-2018]

[4] Avia Semiconductor (2016). 24-Bit Analog-to-Digital Converter (ADC) for Weigh Scales. [En línea]. Disponible en: https://www.mouser.com/ds/2/813/hx711_english-1022875.pdf. [Accedido: 2-jun-2018]

[5] Martinez, Ferreyra, Ramirez, Diagrama de bloques de un sistema de control para automóviles. Universidad Ricardo Palma, Perú. Marzo 2018. 


\section{ANEXOS:}

\section{Costos del proyecto}

Los costos para la realización del prototipo descrito se muestran a continuación en la tabla 2:

Tabla 2. Tabla de costos del proyecto

\begin{tabular}{|c|c|c|}
\hline GASTO & $(\mathrm{S} /)^{2}$ & TOTAL (S/.) \\
\hline BIENES & & \multirow{9}{*}{1650.00} \\
\hline Papel bond y útiles de escritorio. & 70.00 & \\
\hline Sensores: celda de carga y MQ-3. & 400.00 & \\
\hline Componentes para hardware de adquisición y acondicionamiento de señales. & 300.00 & \\
\hline Raspberry Pi 3 B. & 350.00 & \\
\hline Memoria USB de 64 GB. & 80.00 & \\
\hline Cartucho de impresora color y blanco/negro. & 150.00 & \\
\hline Relés vehicular y secundario. & 200.00 & \\
\hline Conmutador de arranque & 100.00 & \\
\hline
\end{tabular}

